\title{
Intrinsic energy is a loop Schur function
}

\author{
ThOMAS LAM* AND PAVLO PYLYAVSKYY ${ }^{\dagger}$
}

\begin{abstract}
We give an explicit subtraction-free formula for the energy function in tensor products of Kirillov-Reshetikhin crystals for symmetric powers of the standard representation of $U_{q}^{\prime}\left(\mathfrak{s} \hat{\mathfrak{l}}_{\mathfrak{n}}\right)$. The energy function is shown to be the tropicalization of a stretched staircase shape loop Schur function. The latter were introduced by the authors in the study of total positivity in loop groups.
\end{abstract}

\section{Introduction}

The intrinsic energy function plays an important role in the path model for affine highest weight crystals [7]. The energy function is also related to the charge statistic of Lascoux-Schützenberger on semistandard tableaux (see [12]), which establishes a relation between one dimensional configuration sums arising in solvable lattice models and Kostka-Foulkes polynomials, cf. $[2,7,8]$.

Let $B=B_{1} \otimes \cdots \otimes B_{m}$ be a tensor product of $U_{q}^{\prime}\left(\hat{\mathfrak{s}}_{\mathfrak{n}}\right)$ Kirillov-Reshetikhin crystals, where each $B_{i}$ is the crystal for a symmetric power of the standard representation. We identify $B_{i}$ with the semistandard Young tableaux with row shape, filled with the numbers $1,2, \ldots, n$. Let $b=b_{1} \otimes \cdots \otimes b_{m} \in B$, and write $x_{i}^{(r+i-1)}$ for the number of $r$ 's in $b_{i}$. The upper index $(r+i-1)$ is to be considered as an element of $\mathbb{Z} / n \mathbb{Z}$. Our main result is the following formula for the intrinsic energy function $\bar{D}_{B}$ of $B$.

Let $\delta_{t}=(t, t-1, \ldots, 1)$ denote the staircase shape of side-length $t$.

Theorem 1.1. We have

$$
\bar{D}_{B}(b)=\min _{T}\left\{\sum_{(i, j) \in(n-1) \delta_{m-1}} x_{T(i, j)}^{(i-j)}\right\},
$$

arXiv: 1003.3948

${ }^{*}$ T.L. was supported by NSF grant DMS-0652641 and DMS-0901111, and by a Sloan Fellowship.

${ }^{\dagger}$ P.P. was supported by NSF grant DMS-0757165. 
where the minimum is over all semistandard tableaux $T$ of shape $(n-1) \delta_{m-1}$, and entries in $1,2, \ldots, m$.

In the physical interpretation, each $b_{i}$ represents a particle, and the intrinsic energy function $\bar{D}_{B}(b)$ is defined as the sum of $\left(\begin{array}{c}m \\ 2\end{array}\right)$ local energies of interactions of particles. Theorem 1.1 thus has the following interpretation: each tableau $T$ encodes a way for $m$ particles to interact simultaneously, and intrinsic energy is equal to the minimum of these.

In [10], motivated by the study of total positivity for loop groups, we introduced a generalization of the ring of symmetric functions, called loop symmetric functions and denoted LSym. In particular, we defined distinguished elements of LSym called loop Schur functions (see Section 2). It is shown in [10] that the algebra homomorphisms from LSym to $\mathbb{R}$ taking nonnegative values on (skew) loop Schur functions are in bijection with totally nonnegative elements of the formal loop group.

Recall that the tropicalization of a subtraction-free polynomial $f$, is obtained by replacing multiplication by addition, and replacing addition by taking minimums. Theorem 1.1 is equivalent to

Theorem 1.2. The function $\bar{D}_{B}$ is the tropicalization of the loop Schur function $s_{(n-1) \delta_{m-1}}^{(0)}$ in the variables $\left\{\mathrm{x}_{i}^{(s)}\right\}$.

Theorem 1.2 is an immediate consequence of Theorems 2.5 and 3.2 below.

Theorems 1.1 and 1.2 are canonical in the sense that they correspond to the monomial expansion of a polynomial. That a piecewise-linear expression for $\bar{D}_{B}(b)$ exists is already clear from the literature. However, the fact that a subtraction-free formula exists (or equivalently the rational version $\overline{\mathrm{D}}_{B}(b)$ of energy is a polynomial with positive coefficients) is not apparent from the definition of $\bar{D}_{B}(b)$, even though the latter takes nonnegative values. (See also Remark 3.)

Example 1. Let $n=2$ and $m=3$. Then

$$
\begin{aligned}
& \bar{D}_{B}(b)=\min \left(x_{1}^{(1)}+x_{1}^{(2)}+x_{2}^{(1)}, x_{2}^{(1)}+x_{1}^{(2)}+x_{2}^{(1)}, x_{3}^{(1)}+x_{1}^{(2)}+x_{2}^{(1)},\right. \\
& x_{1}^{(1)}+x_{1}^{(2)}+x_{3}^{(1)}, x_{2}^{(1)}+x_{1}^{(2)}+x_{3}^{(1)}, x_{3}^{(1)}+x_{1}^{(2)}+x_{3}^{(1)}, x_{2}^{(1)}+x_{2}^{(2)}+x_{3}^{(1)}, \\
& \left.x_{3}^{(1)}+x_{2}^{(2)}+x_{3}^{(1)}\right)
\end{aligned}
$$

corresponding to the following tableaux of shape $\delta_{2}=(2,1)$ :

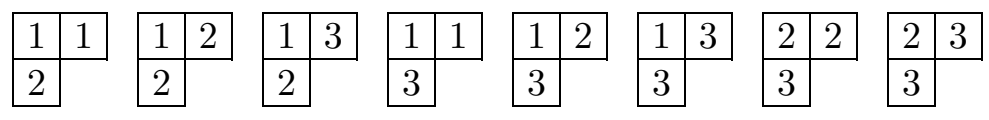


We use in our calculations a birational analogue of the combinatorial $R$-matrix. It was previously studied by Kirillov [6] in the context of the Robinson-Schensted algorithm, by Noumi-Yamada [13, 17] in the context of discrete Painléve systems, by Etingof [3] in the context Yang-Baxter equations, by Berenstein-Kazhdan [1] in the context of geometric crystals, and by the authors [10] in the context of total positivity of loop groups.

\section{Loop symmetric functions}

Fix an integer $n>1$ throughout.

\subsection{Loop Schur functions}

Let $\left(\mathrm{x}_{i}^{(r)}\right)_{1 \leq i \leq m, r \in \mathbb{Z} / n \mathbb{Z}}$ be a rectangular array of variables. We recall from [10] the definition of the ring of loop symmetric functions ${ }^{1}$ in the variables $\mathbf{x}_{i}^{(r)}$, denoted $\operatorname{LSym}_{m}$. A detailed study of loop symmetric functions will appear in [11].

For $k \geq 1$ and $r \in \mathbb{Z} / n \mathbb{Z}$, define the loop elementary symmetric functions and loop complete homogenous symmetric functions by

$$
\begin{aligned}
& e_{k}^{(r)}\left(\mathrm{x}_{1}, \mathrm{x}_{2}, \ldots, \mathrm{x}_{m}\right)=\sum_{1 \leq i_{1}<i_{2}<\cdots<i_{k} \leq m} \mathrm{x}_{i_{1}}^{(r)} \mathrm{x}_{i_{2}}^{(r+1)} \cdots \mathrm{x}_{i_{k}}^{(r+k-1)} \\
& h_{k}^{(r)}\left(\mathrm{x}_{1}, \mathrm{x}_{2}, \ldots, \mathrm{x}_{m}\right)=\sum_{1 \leq i_{1} \leq i_{2} \leq \cdots \leq i_{k} \leq m} \mathrm{x}_{i_{1}}^{(r)} \mathrm{x}_{i_{2}}^{(r-1)} \cdots \mathrm{x}_{i_{k}}^{(r-k+1)} .
\end{aligned}
$$

By convention, $e_{k}^{(r)}=h_{k}^{(r)}=0$ for $k<0$, and $e_{0}^{(r)}=h_{0}^{(r)}=1$. Note that $e_{k}^{(r)}=0$ for $k>m$. We call the upper index the color. When all $n$ colors are identified, that is $\mathrm{x}_{i}^{(s)}=\mathrm{x}_{i}^{\left(s^{\prime}\right)}$ for all $i$ and $s, s^{\prime} \in \mathbb{Z} / n \mathbb{Z}$, these functions specialise to the usual elementary and complete homogenous symmetric functions [16]. We define $\operatorname{LSym}_{m}$ to be the ring generated by the $e_{k}^{(r)}$. Although it is not immediately obvious, the $h_{k}^{(r)}$ lie in $\operatorname{LSym}_{m}$. In fact, both the $e_{k}^{(r)}$ and the $h_{k}^{(r)}$ are instances of distinguished elements of $\operatorname{LSym}_{m}$ called loop Schur functions.

A square $s=(i, j)$ in the $i$-th row and $j$-th column has content $c(s)=$ $i-j$. We caution that our notion of content is the negative of the usual one.

\footnotetext{
${ }^{1}$ In [10], there are two such rings: the ring of whirl loop symmetric functions, and the ring of curl loop symmetric functions. We use the former here. Furthermore, we only use finitely many variables here.
} 
Let $\rho / \nu$ be a skew shape. Recall that a semistandard Young tableaux $T$ with shape $\rho / \nu$ is a filling of each square $s \in \rho / \nu$ with an integer $T(s) \in \mathbb{Z}_{>0}$ so that the rows are weakly-increasing, and columns are increasing. For $r \in \mathbb{Z} / n \mathbb{Z}$, the $r$-weight $\mathrm{x}^{T}$ of a tableaux $T$ is given by $\mathrm{x}^{T}=\prod_{s \in \rho / \nu} \mathrm{x}_{T(s)}^{(c(s)+r)}$.

We shall draw our shapes and tableaux in English notation:

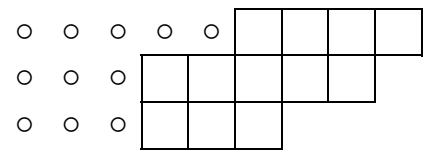

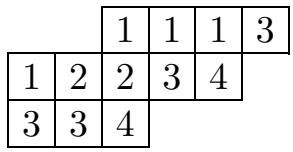

For $n=3$ the 0 -weight of the above tableau is $\left(\mathrm{x}_{1}^{(1)}\right)^{2}\left(\mathrm{x}_{3}^{(1)}\right)^{3} \mathrm{x}_{1}^{(2)} \mathrm{x}_{2}^{(2)} \mathrm{x}_{3}^{(2)} \times$ $\mathrm{x}_{1}^{(3)} \mathrm{x}_{2}^{(3)}\left(\mathrm{x}_{4}^{(3)}\right)^{2}$. We define the loop (skew) Schur function by

$$
s_{\rho / \nu}^{(r)}(\mathrm{x})=\sum_{T} \mathrm{x}^{T}
$$

where the summation is over all semistandard Young tableaux of (skew) shape $\rho / \nu$. We have the following analog of the Jacobi-Trudi formula.

Theorem 2.1 ([10, Theorem 7.6]). We have $s_{\lambda^{\prime} / \mu^{\prime}}^{(r)}=\operatorname{det}\left(e_{\lambda_{i}-\mu_{j}-i+j}^{\left(r-j+\mu_{j}\right)}\right)$.

Proposition 2.2. For any $k>0$ we have

$$
e_{0}^{(r)} h_{k}^{(r-1)}-e_{1}^{(r-1)} h_{k-1}^{(r-2)}+e_{2}^{(r-2)} h_{k-2}^{(r-3)}-\cdots \pm e_{k}^{(r-k)} h_{0}^{(r-k-1)}=0 .
$$

Proof. Let $\rho_{i}$ denote the hook shape $(k-i, 1, \ldots, 1)$ of size $k$. Then $e_{i}^{(r-i)} \times$ $h_{k-i}^{(r-i-1)}=s_{\rho_{k-i}}^{(r-i-1)}+s_{\rho_{k-i+1}}^{(r-i)}$ is the sum of two loop Schur functions (one of which is zero if $i=0$ or $i=k$ ). This holds since any two terms in $e_{i}^{(r-i)}$ and $h_{k-i}^{(r-i-1)}$, viewed as a tableaux of column and row shapes, fit together to give a semistandard tableau of one of the two hook shapes, depending on the entry in their smallest boxes. It is clear that as we sum over all $i$, all the hook shape loop Schur functions cancel out.

\subsection{Staircases}

For $k \geq 1$ and $r \in \mathbb{Z} / n \mathbb{Z}$, define

$$
\tau_{k}^{(r)}\left(\mathrm{x}_{1}, \mathrm{x}_{2}, \ldots, \mathrm{x}_{m}\right)=\sum_{I=\left\{i_{1} \leq i_{2} \leq \cdots \leq i_{k}\right\}} \mathrm{x}_{i_{1}}^{(r)} \mathrm{x}_{i_{2}}^{(r-1)} \cdots \mathrm{x}_{i_{k}}^{(r-k+1)}
$$


where the summation is over multisets $I \subset\{1,2,3, \ldots, m\}$ such that no number occurs more than $n-1$ times. Note that if $k>m(n-1)$ we have $\tau_{k}^{(r)}=0$. It can be shown that $\tau_{k}^{(r)}$ lies in $\operatorname{LSym}_{m}$, but we shall not need it for what follows.

Lemma 2.3. We have

$$
\tau_{k}^{(r)}\left(\mathrm{x}_{1}, \ldots, \mathrm{x}_{m}\right)=\sum_{i=0}^{\infty}(-1)^{i} h_{k-i n}^{(r)} e_{i}\left(\prod_{s \in \mathbb{Z} / n \mathbb{Z}} \mathrm{x}_{1}^{(s)}, \ldots, \prod_{s \in \mathbb{Z} / n \mathbb{Z}} \mathbf{x}_{m}^{(s)}\right),
$$

where the $e_{i}$ in the above formula denotes the usual elementary symmetric function.

Proof. Let $\mathrm{x}_{i_{1}}^{(r)} \mathrm{x}_{i_{2}}^{(r-1)} \cdots \mathrm{x}_{i_{k}}^{(r-k+1)}$ be a term in $h_{k}^{(r)}$. Let $J \subseteq I$ be the set of indexes which occur in $I=\left\{i_{1} \leq i_{2} \leq \cdots \leq i_{k}\right\}$ more than $n-1$ times. Then the coefficient of this term on the right is equal to $\sum_{K \subseteq J}(-1)^{|K|}$. This is equal to 1 if $|J|=0$ and to 0 otherwise.

Lemma 2.4. For each $k$, we have

$$
\sum_{i=0}^{\infty}(-1)^{i} e_{i}^{(r-i)}\left(\mathrm{x}_{1}, \ldots, \mathrm{x}_{m}\right) \tau_{k-i}^{(r-i-1)}\left(\mathrm{x}_{1}, \ldots, \mathrm{x}_{m}\right)=0 .
$$

Proof. Follows immediately from Proposition 2.2 and Lemma 2.3.

Define $\sigma_{k}^{(r)}\left(\mathrm{x}_{1}, \mathrm{x}_{2}, \ldots, \mathrm{x}_{m}\right)=\sum_{i=0}^{k} \mathrm{x}_{1}^{(r)} \mathrm{x}_{1}^{(r-1)} \cdots \mathrm{x}_{1}^{(r-i+1)} \tau_{k-i}^{(r-i)}\left(\mathrm{x}_{2}, \ldots, \mathrm{x}_{m}\right)$.

Theorem 2.5. For $m \geq 2$ and $r \in \mathbb{Z} / n \mathbb{Z}$, we have

$$
\begin{aligned}
& s_{(n-1) \delta_{m-1}}^{(r)}\left(\mathrm{x}_{1}, \mathrm{x}_{2}, \ldots, \mathrm{x}_{m}\right) \\
& =\sigma_{(n-1)(m-1)}^{(r)}\left(\mathrm{x}_{1}, \ldots, \mathrm{x}_{m}\right) \sigma_{(n-1)(m-2)}^{(r+1)}\left(\mathrm{x}_{2}, \ldots, \mathrm{x}_{m}\right) \cdots \sigma_{(n-1)}^{(r+m-2)}\left(\mathrm{x}_{m-1}, \mathrm{x}_{m}\right) .
\end{aligned}
$$

Remark 1. When all the colors are identified, that is, $\mathbf{x}_{i}^{(s)}=\mathrm{x}_{i}^{\left(s^{\prime}\right)}$ for all $i$ and $s, s^{\prime} \in \mathbb{Z} / n \mathbb{Z}$, Theorem 2.5 is a coarsening of a result of Jucis [5], see also [16, Ex. 7.30].

It is clear that Theorem 2.5 holds for $m=2$, for then it states that $s_{n-1}^{(r)}\left(\mathrm{x}_{1}, \mathrm{x}_{2}\right)=\sigma_{n-1}^{(r)}\left(\mathrm{x}_{1}, \mathrm{x}_{2}\right)$. We shall prove Theorem 2.5 in Section 4. 


\section{Affine crystals}

\section{1. $R$-matrix}

We shall use [15] as our main reference for affine crystals.

Recall that a Kirillov-Reshetikhin crystal of $U_{q}^{\prime}\left(\mathfrak{s}_{\mathfrak{n}}\right)$ is the crystal graph corresponding to the highest weight module with highest weight proportional to one of the fundamental weights. An affine crystal is the tensor product of several Kirillov-Reshetikhin crystals. We shall restrict our attention to the set $\mathfrak{C}$ of affine crystals that are tensor products of symmetric powers of the standard representation. Each element $b \in B$ of such a symmetric power can be identified with a single row semistandard tableau in the alphabet $1, \ldots, n$.

If $B_{1}, B_{2}$ are Kirillov-Reshetikhin crystals, the combinatorial $R$-matrix is the unique isomorphism $R_{B_{1}, B_{2}}: B_{1} \otimes B_{2} \rightarrow B_{2} \otimes B_{1}$ of affine crystals. It is known that the combinatorial $R$-matrices generate an action of $S_{m}$ on $B_{1} \otimes \cdots \otimes B_{m}$.

The combinatorial $R$-matrix has a convenient interpretation [15] in terms of semistandard tableaux and the jeu de taquin algorithm [16]. Let $b_{1} \otimes b_{2}$ be an element of $B_{1} \otimes B_{2}$. Then $R_{B_{1}, B_{2}}\left(b_{1} \otimes b_{2}\right)=c_{1} \otimes c_{2} \in B_{2} \otimes B_{1}$ where $c_{1}, c_{2}$ are the unique pair of row shaped tableaux which jeu de taquin to the same tableau that $b_{1}$ and $b_{2}$ jeu de taquin to, as follows:

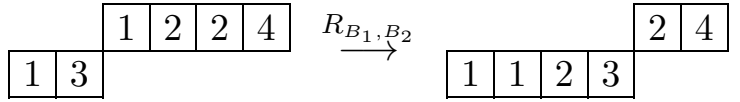

$$
\begin{aligned}
& \text { since both jeu de taquin to } \begin{array}{|l|l|l|l|l|}
\hline 1 & 1 & 2 & 2 & 4 \\
\hline 3 & \multicolumn{7}{|l}{} \\
\cline { 2 - 3 } & \multicolumn{1}{|l}{}
\end{array}
\end{aligned}
$$

The action of the combinatorial $R$-matrix can be explicitly described as follows (see [4]). Let $R_{B_{1}, B_{2}}\left(b_{1} \otimes b_{2}\right)=c_{1} \otimes c_{2}$ and let $\bar{x}_{1}^{(r)}, \bar{x}_{2}^{(r)}, s_{1}\left(\bar{x}_{1}^{(r)}\right)$, $s_{1}\left(\bar{x}_{2}^{(r)}\right)$ be the number of boxes filled with $r$-s in $b_{1}, b_{2}, c_{1}, c_{2}$ respectively, $r=1, \ldots, n$. Then

$$
\begin{aligned}
& s_{1}\left(\bar{x}_{1}^{(r)}\right)=\bar{x}_{2}^{(r)}+\bar{\kappa}_{r+1}\left(b_{1}, b_{2}\right)-\bar{\kappa}_{r}\left(b_{1}, b_{2}\right) \text { and } \\
& s_{1}\left(\bar{x}_{2}^{(r)}\right)=\bar{x}_{1}^{(r)}+\bar{\kappa}_{r}\left(b_{1}, b_{2}\right)-\bar{\kappa}_{r+1}\left(b_{1}, b_{2}\right)
\end{aligned}
$$

where

$$
\bar{\kappa}_{r}\left(b_{1}, b_{2}\right)=\min _{0 \leq s \leq n-1}\left(\sum_{t=1}^{s} \bar{x}_{2}^{(r+t-1)}+\sum_{t=s+1}^{n-1} \bar{x}_{1}^{(r+t)}\right)
$$

and the indexes are taken in $\mathbb{Z} / n \mathbb{Z}$. 
Example 2. In the example above $\left(\bar{x}_{1}^{(1)}, \bar{x}_{1}^{(2)}, \bar{x}_{1}^{(3)}, \bar{x}_{1}^{(4)}\right)=(1,0,1,0),\left(\bar{x}_{2}^{(1)}\right.$, $\left.\bar{x}_{2}^{(2)}, \bar{x}_{2}^{(3)}, \bar{x}_{2}^{(4)}\right)=(1,2,0,1), \bar{\kappa}_{1}\left(b_{1}, b_{2}\right)=\min (1,2,3,3)=1, \bar{\kappa}_{2}\left(b_{1}, b_{2}\right)=$ $\min (2,3,3,3)=2$ and $s_{1}\left(\bar{x}_{1}^{(1)}\right)=1+2-1=2$.

\subsection{Intrinsic energy function}

In [7] an important function $\bar{H}_{B, B^{\prime}}: B \otimes B^{\prime} \rightarrow \mathbb{Z}$ called local coenergy is defined for a tensor product of two affine crystals. If $B$ and $B^{\prime}$ are KirillovReshetikhin crystals, local coenergy has the following simple description in terms of tableaux $[9,15]$. Given an element $b \otimes b^{\prime}$ in $B \otimes B^{\prime} \in \mathfrak{C}$, form a two-row semistandard tableaux from $b$ and $b^{\prime}$ as above. After that, measure the maximal number of cells one can slide the top row to the left so that we still have a valid semistandard tableau. This maximal number of cells is the value of $\bar{H}_{B, B^{\prime}}\left(b, b^{\prime}\right)$.

Example 3. For

$$
\begin{array}{|l|l|l|l|l|l|l|l|l|}
\cline { 2 - 7 } \multicolumn{2}{c|}{} & 1 & 2 & 3 & 3 & 4 \\
\hline
\end{array}
$$

the local coenergy is 3 , because

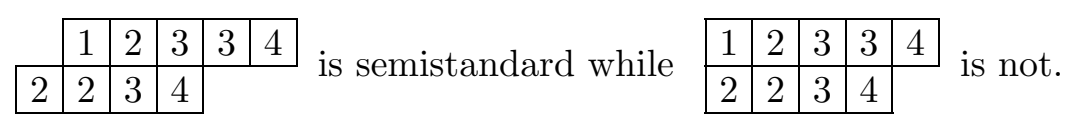

It is easy to see that $\bar{H}_{B, B^{\prime}}\left(b, b^{\prime}\right)=\bar{\kappa}_{1}\left(b, b^{\prime}\right)$, since each term $\sum_{t=1}^{s} \bar{x}_{2}^{(t)}+$ $\sum_{t=s+1}^{n-1} \bar{x}_{1}^{(1+t)}$ is exactly the number of cells for which the boxes with $(s+1)$-s in them would allow sliding. The local coenergy is known to remain the same under the action of $R$-matrix: $\bar{H}_{B^{\prime}, B}=\bar{H}_{B, B^{\prime}} \circ R_{B^{\prime}, B}$.

We now define the intrinsic energy function $\bar{D}_{B}: B \rightarrow \mathbb{Z}([7])$ of an affine crystal $B \in \mathfrak{C}$, following [14]. For Kirillov-Reshetikhin crystals, the energy is zero. Let $b=b_{1} \otimes b_{2} \otimes \cdots \otimes b_{m}$ be an element of an $m$-fold tensor product $B=B_{1} \otimes B_{2} \otimes \cdots \otimes B_{m} \in \mathfrak{C}$. We define the intrinsic energy $\bar{D}_{B}(b)$ to be

$$
\bar{D}_{B}(b)=\sum_{1 \leq i<j \leq m} \bar{H}_{B_{i}, B_{j}}\left(s_{i} s_{i+1} \cdots s_{j-2}\left(b_{j-1}\right) \otimes b_{j}\right) .
$$

Although not obvious from this definition, intrinsic energy is preserved by the $R$-action. 
Example 4. Let us compute the intrinsic energy of the following element $b=b_{1} \otimes b_{2} \otimes b_{3}$.

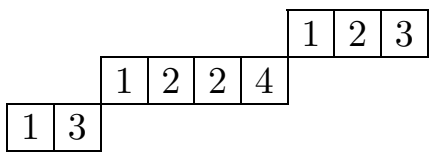

We measure $\bar{H}\left(b_{1}, b_{2}\right)=1, \bar{H}\left(b_{2}, b_{3}\right)=2$. We also have seen above the result of applying $R_{B_{1}, B_{2}}$ to the first two tensor factors, which allows us to measure $\bar{H}\left(s_{1}\left(b_{2}\right), b_{3}\right)=2$. Therefore $\bar{D}_{B}(b)=1+2+2=5$.
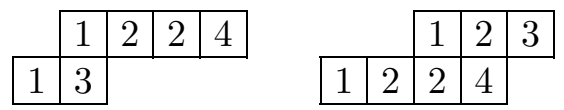

\begin{tabular}{|l|l|l|}
\hline 1 & 2 & 3 \\
\hline 2 & 4 & \multicolumn{1}{|c}{} \\
\cline { 1 - 2 }
\end{tabular}

\subsection{Product (summation) formula for intrinsic energy}

In this section, we switch from piecewise linear functions to rational functions. The two worlds are connected via tropicalization: if $f$ is a subtractionfree polynomial, we let $\operatorname{trop}(f)$ denote the tropicalization of $f$, obtained by replacing addition by minimum, and multiplication by addition.

We are given a rectangular array of variables $\overline{\mathrm{x}}_{i}^{(r)}, i=1, \ldots, m, r \in$ $\mathbb{Z} / n \mathbb{Z}$, with columns $b_{i}=\left(\overline{\mathrm{x}}_{i}^{(1)}, \overline{\mathrm{x}}_{i}^{(2)}, \ldots, \overline{\mathrm{x}}_{i}^{(n)}\right)$. It is very convenient to make the following change of variables: $\mathrm{x}_{i}^{(r)}=\overline{\mathrm{x}}_{i}^{(r+1-i)}$. Define

$$
\kappa_{r}\left(b_{j}, b_{j+1}\right)=\sum_{s=0}^{n-1}\left(\prod_{t=1}^{s} \mathrm{x}_{j+1}^{(r+t)} \prod_{t=s+1}^{n-1} \mathrm{x}_{j}^{(r+t)}\right)
$$

so that $\operatorname{trop}\left(\kappa_{r}\left(b_{j}, b_{j+1}\right)\right)=\bar{\kappa}_{r-j+1}\left(b_{j}, b_{j+1}\right)$.

In the variables $\mathrm{x}_{i}^{(r)}$, the birational $R$-matrix acts (see [17, Proposition 3.1$]^{2}$ ) via algebra isomorphisms $s_{1}, s_{2}, \ldots, s_{m-1}$ of the field of rational functions in $\left\{\mathrm{x}_{i}^{(r)}\right\}$, given by

$$
s_{j}\left(\mathrm{x}_{j}^{(r)}\right)=\frac{\mathrm{x}_{j+1}^{(r+1)} \kappa_{r+1}\left(b_{j}, b_{j+1}\right)}{\kappa_{r}\left(b_{j}, b_{j+1}\right)} \quad \text { and } \quad s_{j}\left(\mathrm{x}_{j+1}^{(r)}\right)=\frac{\mathrm{x}_{j}^{(r-1)} \kappa_{r-1}\left(b_{j}, b_{j+1}\right)}{\kappa_{r}\left(b_{j}, b_{j+1}\right)}
$$

and $s_{j}\left(\mathrm{x}_{k}^{(r)}\right)=\mathrm{x}_{k}^{(r)}$ for $k \neq j, j+1$. We also have $\overline{\mathrm{H}}\left(b_{j} \otimes b_{j+1}\right)=\kappa_{j}\left(b_{j}, b_{j+1}\right)$, the rational analogue of the local coenergy. We let $\overline{\mathrm{D}}_{B}$ denote the rational

${ }^{2}$ Our variables $\overline{\mathbf{x}}_{i}^{(r)}$ are nearly the same as Yamada's $x_{r}^{i}$, differing by a reversal of the orientation of the circle. 
analogue of the intrinsic energy function, so that $\operatorname{trop}\left(\overline{\mathrm{D}}_{B}\right)=\bar{D}_{B}$. The main result of this section is a product formula for $\overline{\mathrm{D}}_{B}$. We remark that Kirillov [6] has also studied the rational functions $\overline{\mathrm{H}}$ and $\overline{\mathrm{D}}_{B}$.

Lemma 3.1. Suppose $1 \leq i<j \leq m$. Then

$$
\kappa_{r}\left(s_{i} s_{i+1} \cdots s_{j-2}\left(b_{j-1}\right), b_{j}\right)=\frac{\sigma_{(n-1)(j-i)}^{(r-j+i)}\left(\mathrm{x}_{i}, \mathrm{x}_{i+1}, \ldots, \mathrm{x}_{j}\right)}{\sigma_{(n-1)(j-i-1)}^{(r-j+i)}\left(\mathrm{x}_{i}, \mathrm{x}_{i+1}, \ldots, \mathrm{x}_{j-1}\right)}
$$

and

$$
\begin{aligned}
s_{i} s_{i+1} \cdots s_{j-1}\left(\mathrm{x}_{j}^{(r)}\right)= & \frac{\mathrm{x}_{i}^{(r-j+i)} \sigma_{(n-1)(j-i)}^{(r-j+i-1)}\left(\mathrm{x}_{i}, \mathrm{x}_{i+1}, \ldots, \mathrm{x}_{j}\right)}{\sigma_{(n-1)(j-i)}^{(r-j+i)}\left(\mathrm{x}_{i}, \mathrm{x}_{i+1}, \ldots, \mathrm{x}_{j}\right)} \\
& =\frac{\sigma_{(n-1)(j-i)+1}^{(r-j+i)}\left(\mathrm{x}_{i}, \mathrm{x}_{i+1}, \ldots, \mathrm{x}_{j}\right)}{\sigma_{(n-1)(j-i)}^{(r-j+i)}\left(\mathrm{x}_{i}, \mathrm{x}_{i+1}, \ldots, \mathrm{x}_{j}\right)}
\end{aligned}
$$

Proof. We prove the two statements in parallel by induction on $j-i$. For $j-i=1$ they coincide with the formulae for the $\kappa_{r}$ and the $R$-action of $s_{i}$. By the induction assumption

$$
s_{i} s_{i+1} \cdots s_{j-2}\left(\mathrm{x}_{j-1}^{(r)}\right)=\frac{\mathrm{x}_{i}^{(r-j+i+1)} \sigma_{(n-1)(j-i-1)}^{(r-j+i)}\left(\mathrm{x}_{i}, \mathrm{x}_{i+1}, \ldots, \mathrm{x}_{j-1}\right)}{\sigma_{(n-1)(j-1-i)}^{(r-j+i+1)}\left(\mathrm{x}_{i}, \mathrm{x}_{i+1}, \ldots, \mathrm{x}_{j-1}\right)} .
$$

Therefore

$$
\begin{aligned}
& \kappa_{r}\left(s_{i} s_{i+1} \cdots s_{j-2}\left(b_{j-1}\right), b_{j}\right) \\
& =\sum_{s=0}^{n-1}\left(\prod_{t=1}^{s} \mathbf{x}_{j}^{(r+t)} \prod_{t=s+1}^{n-1} s_{i} s_{i+1} \cdots s_{j-2}\left(\mathbf{x}_{j-1}^{(r+t)}\right)\right) \\
& =\sum_{s=0}^{n-1} \frac{\prod_{t=s-j+i+2}^{n-j+i} \mathbf{x}_{i}^{(r+t)} \sigma_{(n-1)(j-i-1)}^{(r+s-j+i+1)}\left(\mathrm{x}_{i}, \mathrm{x}_{i+1}, \ldots, \mathrm{x}_{j-1}\right) \prod_{t=1}^{s} \mathbf{x}_{j}^{(r+t)}}{\sigma_{(n-1)(j-i-1)}^{(r-j+i)}\left(\mathrm{x}_{i}, \mathrm{x}_{i+1}, \ldots, \mathrm{x}_{j-1}\right)} \\
& =\frac{\sigma_{(n-1)(j-i)}^{(r-j+i)}\left(\mathrm{x}_{i}, \mathrm{x}_{i+1}, \ldots, \mathrm{x}_{j}\right)}{\sigma_{(n-1)(j-i-1)}^{(r-j+i)}\left(\mathrm{x}_{i}, \mathrm{x}_{i+1}, \ldots, \mathrm{x}_{j-1}\right)}
\end{aligned}
$$

The last equality holds because in a term of $\sigma_{(n-1)(j-i)}^{(r-j+i)}\left(\mathrm{x}_{i}, \mathrm{x}_{i+1}, \ldots, \mathrm{x}_{j}\right)$ the number of the $\mathrm{x}_{j}^{(t)}$-s is at most $n-1$, while the number of the $\mathbf{x}_{i}^{(t)}$-s and the $\mathrm{x}_{j}^{(t)}$-s together should be at least $n-1$. 
Now we can also prove the second claim, since

$$
\begin{aligned}
s_{i} s_{i+1} \cdots s_{j-1}\left(\mathrm{x}_{j}^{(r)}\right) & \\
= & \frac{s_{i} s_{i+1} \cdots s_{j-2}\left(\mathrm{x}_{j-1}^{(r-1)}\right) \kappa_{r-1}\left(s_{i} s_{i+1} \cdots s_{j-2}\left(b_{j-1}\right), b_{j}\right)}{\kappa_{r}\left(s_{i} s_{i+1} \cdots s_{j-2}\left(b_{j-1}\right), b_{j}\right)} \\
= & \frac{\mathbf{x}_{i}^{(r-j+i)} \sigma_{(n-1)(j-i-1)}^{(r-j+i-1)}\left(\mathrm{x}_{i}, \ldots, \mathrm{x}_{j-1}\right)}{\sigma_{(n-1)(j-1-i)}^{(r-j+i)}\left(\mathrm{x}_{i}, \ldots, \mathrm{x}_{j-1}\right)} \frac{\sigma_{(n-1)(j-i)}^{(r-j+i-1)}\left(\mathrm{x}_{i}, \ldots, \mathrm{x}_{j}\right)}{\sigma_{(n-1)(j-i-1)}^{(r-j+i-1)}\left(\mathrm{x}_{i}, \ldots, \mathrm{x}_{j-1}\right)} \\
& \times \frac{\sigma_{(n-1)(j-i-1)}^{(r-j+i)}\left(\mathrm{x}_{i}, \ldots, \mathrm{x}_{j-1}\right)}{\sigma_{(n-1)(j-i)}^{(r-j+i)}\left(\mathrm{x}_{i}, \ldots, \mathrm{x}_{j}\right)} \\
= & \frac{\mathrm{x}_{i}^{(r-j+i)} \sigma_{(n-1)(j-i)}^{(r-j+i-1)}\left(\mathrm{x}_{i}, \ldots, \mathrm{x}_{j}\right)}{\sigma_{(n-1)(j-i)}^{(r-j+i)}\left(\mathrm{x}_{i}, \ldots, \mathrm{x}_{j}\right)}
\end{aligned}
$$

Theorem 3.2. We have

$\overline{\mathrm{D}}_{B}(b)=\sigma_{(n-1)(m-1)}^{(n)}\left(\mathrm{x}_{1}, \ldots, \mathrm{x}_{m}\right) \sigma_{(n-1)(m-2)}^{(1)}\left(\mathrm{x}_{2}, \ldots, \mathrm{x}_{m}\right) \cdots \sigma_{(n-1)}^{(m-2)}\left(\mathrm{x}_{m-1}, \mathrm{x}_{m}\right)$.

Proof. The result follows from Lemma 3.1 and (1).

Remark 2. Comparing [12, Theorem 4.2] with Lemma 3.1 and Theorem 3.2 one can see that the tropicalization of the $\sigma_{(n-1)(m-1-i)}^{(i)}\left(\mathbf{x}_{i+1}, \ldots, \mathbf{x}_{m}\right)$ is essentially the index ind $(m-i)$ in the index decomposition of charge in [12].

Remark 3. Theorem 3.2 gives the irreducible factorization of $\overline{\mathrm{D}}_{B}(b)$ (whereas Theorem 1.2 gives the monomial expansion). To see this, one first notes that $\sigma_{(n-1)}^{(r)}\left(\mathrm{x}_{1}, \mathrm{x}_{2}\right)$ has a unique monomial which involves $\mathrm{x}_{2}^{(r)}$, from which one deduces the irreducibility. Now suppose that $\sigma_{(n-1)(m-1)}^{(r)}\left(\mathrm{x}_{1}, \mathrm{x}_{2}, \ldots, \mathrm{x}_{m}\right)$ factorizes non-trivially as the product $f g$. We may write

$$
f=a \mathrm{x}_{m}^{(r-(n-1)(m-2))}+b
$$

and $g=c$ as polynomials in $\mathbf{x}_{m}^{(r-(n-1)(m-2))}$, where $a, b, c$ do not involve $\mathbf{x}_{m}^{(r-(n-1)(m-2))}$. One verifies that none of the variables $\mathbf{x}_{m}^{(s)}$ divide $\sigma_{(n-1)(m-1)}^{(r)}\left(\mathbf{x}_{1}, \mathbf{x}_{2}, \ldots, \mathbf{x}_{m}\right)$ and every monomial which contains $\mathbf{x}_{m}^{(r-(n-1)(m-2))}$ is divisible by the product

$$
\mathbf{x}_{m}^{(r-(n-1)(m-2))} \mathbf{x}_{m}^{(r-(n-1)(m-2)-1)} \cdots \mathbf{x}_{m}^{(r-(n-1)(m-1)+1)}
$$


Thus we have $f=a^{\prime} \mathbf{x}_{m}^{(r-(n-1)(m-2))} \mathbf{x}_{m}^{(r-(n-1)(m-2)-1)} \cdots \mathbf{x}_{m}^{(r-(n-1)(m-1)+1)}+$ $b$, where $a^{\prime}$ is a polynomial not involving any $\mathbf{x}_{m}^{(s)}$. It is easy to see that $a^{\prime}$ cannot be a unit. But we then have a non-trivial factorization $a^{\prime} c=$ $\sigma_{(n-1)(m-2)}^{(r)}\left(\mathrm{x}_{1}, \ldots, \mathrm{x}_{m-1}\right)$, and we may proceed by induction.

\section{Proof of Theorem 2.5}

We let $A_{m}$ denote the Jacobi-Trudi matrix for the dilated staircase Schur funtion $s_{(n-1) \delta_{m-1}}^{r}$. By adding extra columns of size 0 to $(n-1) \delta_{m-1}$ we may assume that $A_{m}$ is a $n a \times n a$ matrix. (Specifically, $a=\lceil(n-1)(m-1) / n\rceil$.)

Example 5. For $n=3$ we have

$$
A_{4}=\left(\begin{array}{cccccc}
e_{3}^{(r)} & e_{4}^{(r-1)} & & & & \\
e_{2}^{(r)} & e_{3}^{(r-1)} & e_{4}^{(r-2)} & & & \\
e_{0}^{(r)} & e_{1}^{(r-1)} & e_{2}^{(r-2)} & e_{3}^{(r)} & e_{4}^{(r-1)} & \\
& e_{0}^{(r-1)} & e_{1}^{(r-2)} & e_{2}^{(r)} & e_{3}^{(r-1)} & e_{4}^{(r-2)} \\
& & & e_{0}^{(r)} & e_{1}^{(r-1)} & e_{2}^{(r-2)} \\
& & & & e_{0}^{(r-1)} & e_{1}^{(r-2)}
\end{array}\right)
$$

Lemma 4.1. Suppose $n<i \leq n a$. Then column $i$ of $A_{m}$ is obtained from column $i-n$ by shifting the non-zero entries down by $n-1$.

Let $B_{m}$ denote the $n(a+1)-1 \times n(a+1)$ matrix obtained by adding $n$ extra columns to $A_{m}$, so that Lemma 4.1 is still true.

Example 6. For $n=3$ we have

$$
B_{4}=\left(\begin{array}{cccccccccc}
e_{3}^{(r)} & e_{4}^{(r-1)} & & & & & & & \\
e_{2}^{(r)} & e_{3}^{(r-1)} & e_{4}^{(r-2)} & & & & & & \\
e_{0}^{(r)} & e_{1}^{(r-1)} & e_{2}^{(r-2)} & e_{3}^{(r)} & e_{4}^{(r-1)} & & & & \\
& e_{0}^{(r-1)} & e_{1}^{(r-2)} & e_{2}^{(r)} & e_{3}^{(r-1)} & e_{4}^{(r-2)} & & & \\
& & & e_{0}^{(r)} & e_{1}^{(r-1)} & e_{2}^{(r-2)} & e_{3}^{(r)} & e_{4}^{(r-1)} & \\
& & & & e_{0}^{(r-1)} & e_{1}^{(r-2)} & e_{2}^{(r)} & e_{3}^{(r-1)} & e_{4}^{(r-2)} \\
& & & & & & e_{0}^{(r)} & e_{1}^{(r-1)} & e_{2}^{(r-2)} \\
& & & & & & & e_{0}^{(r-1)} & e_{1}^{(r-2)}
\end{array}\right)
$$

Let

$$
\mathcal{T}=\left(\tau_{(n-1) m}^{(r-1)},-\tau_{(n-1) m-1}^{(r-2)}, \ldots, \pm \tau_{(n-1) m-n(a+1)+1}^{(r-n(a+1)+1)}\right)
$$

be a column vector with components in LSym. 
Proposition 4.2. The vector $B_{m} \cdot \mathcal{T}$ is the zero vector.

Proof. It follows immediately from Lemma 2.4, and the fact that $\tau_{k}^{(s)}=0$ as long as $k>(n-1) m$.

Let $B_{i, m}$ be the square matrix obtained from $B_{m}$ by removing the $i$-th column. It is easy to see that $\operatorname{det}\left(B_{i, m}\right)=s_{(n-1) \delta_{m} /(i-1)}^{(r-1)}$ is a loop skew Schur function.

Proposition 4.3. We have $\operatorname{det}\left(B_{i, m}\right)=\tau_{(n-1) m-i+1}^{(r-i)} \operatorname{det}\left(A_{m}\right)$.

Proof. Since $\operatorname{det}\left(B_{i, m}\right)=s_{(n-1) \delta_{m} /(i-1)}^{(r-1)}$ is never the zero polynomial in the $e_{i}^{(s)}$, we deduce that considered as a matrix with ceofficients in the field $\operatorname{Frac}\left(\mathrm{x}_{i}^{(s)}\right)$, the matrix $B_{m}$ has maximal rank. There is thus, up to scaling, a unique solution to the equation $B_{m} \cdot v=0$. By expanding the determinant of the matrix obtained from $B_{m}$ by repeating a row, it is easy to see that $v=\left(\operatorname{det}\left(B_{1, m}\right),-\operatorname{det}\left(B_{2, m}\right), \ldots, \pm \operatorname{det}\left(B_{n a+n, m}\right)\right)$ is a solution. But by Proposition 4.2 so is $\mathcal{T}$. Thus the two vectors are proportional, and it remains to check that the scaling coefficient is $\operatorname{det}\left(A_{m}\right)$. We have $\operatorname{det}\left(B_{1, m}\right)=e_{m}^{(r-1)} \cdots e_{m}^{(r-n+1)} \operatorname{det}\left(A_{m}\right)$, while at the same time $\tau_{(n-1) m}^{(r-1)}=e_{m}^{(r-1)} \cdots e_{m}^{(r-n+1)}$, and the statement follows.

Proof of Theorem 2.5. We have already verified the case $m=2$, so we suppose that $m>2$, and by induction on $m$ that

$$
\begin{aligned}
& s_{(n-1) \delta_{m-1}\left(\mathrm{x}_{2}, \mathrm{x}_{3}, \ldots, \mathrm{x}_{m+1}\right)}^{(s+1)} \\
& \quad=\sigma_{(n-1)(m-1)}^{(s+1)}\left(\mathrm{x}_{2}, \ldots, \mathrm{x}_{m+1}\right) \sigma_{(n-1)(m-2)}^{(s+2)} \\
& \quad \times\left(\mathrm{x}_{3}, \ldots, \mathrm{x}_{m+1}\right) \cdots \sigma_{(n-1)}^{(s+m-1)}\left(\mathrm{x}_{m}, \mathrm{x}_{m+1}\right)
\end{aligned}
$$

for every $s \in \mathbb{Z} / n \mathbb{Z}$. We calculate that

$$
\begin{aligned}
& s_{(n-1) \delta_{m}}^{(r-1)}\left(\mathrm{x}_{1}, \mathrm{x}_{2}, \ldots, \mathrm{x}_{m+1}\right) \\
& \quad=\sum_{i=0}^{(n-1) m} \mathrm{x}_{1}^{(r-1)} \mathrm{x}_{1}^{(r-2)} \cdots \mathrm{x}_{1}^{(r-i)} s_{(n-1) \delta_{m} /(i)}^{(r-1)}\left(\mathrm{x}_{2}, \mathrm{x}_{3}, \ldots, \mathrm{x}_{m+1}\right) \\
& \quad=\sum_{i=0}^{(n-1) m} \mathrm{x}_{1}^{(r-1)} \mathrm{x}_{1}^{(r-2)} \cdots \mathrm{x}_{1}^{(r-i)} \operatorname{det}\left(B_{i, m}\right)\left(\mathrm{x}_{2}, \mathrm{x}_{3}, \ldots, \mathrm{x}_{m+1}\right)
\end{aligned}
$$




$$
\begin{aligned}
= & \left(\sum_{i=0}^{(n-1) m} \mathrm{x}_{1}^{(r-1)} \mathrm{x}_{1}^{(r-2)} \cdots \mathrm{x}_{1}^{(r-i)} \tau_{(n-1) m-i+1}^{(r-i-1)}\left(\mathrm{x}_{2}, \mathrm{x}_{3}, \ldots, \mathrm{x}_{m+1}\right)\right) \\
& \times s_{(n-1) \delta_{m-1}}^{(r)}\left(\mathrm{x}_{2}, \mathrm{x}_{3}, \ldots, \mathbf{x}_{m+1}\right) \\
= & \sigma_{(n-1) m}^{(r-1)}\left(\mathrm{x}_{1}, \mathrm{x}_{2}, \ldots, \mathbf{x}_{m+1}\right) s_{(n-1) \delta_{m-1}}^{(r)}\left(\mathrm{x}_{2}, \mathrm{x}_{3}, \ldots, \mathbf{x}_{m+1}\right)
\end{aligned}
$$

where in the first equality we used the tableau definition of $s_{(n-1) \delta_{m}}^{(r)}$, and in the penultimate equality we used Proposition 4.3.

Remark 4. It is clear from the theory developed in [10] that loop Schur functions are invariants of the action of the symmetric group $S_{m}$ via the birational $R$-action. Thus we have demonstrated directly that the energy function is an invariant of this action. This property is not obvious from the definition we use.

\section{References}

[1] A. Berenstein and D. Kazhdan (2007). Geometric and unipotent crystals. II. From unipotent bicrystals to crystal bases. Quantum Groups 433 13-88. MR2349617

[2] E. Date, M. Jimbo, A. Kuniba, T. Miwa, and M. Okado (1989). One dimensional configuration sums in vertex models and affine Lie algebra characters. LMP 17 69-77. MR0990586

[3] P. Etingof (2003). Geometric crystals and set-theoretical solutions to the quantum Yang-Baxter equation. Comm. Algebra 31 1961-1973. MR1972900

[4] G. Hatayama, K. Hikami, R. Inoue, A. Kuniba, T. Takagi, and T. Tokihiro (2001). The $A_{M}^{(1)}$ automata related to crystals of symmetric tensors. J. Math. Phys. 42 274-308. MR1808778

[5] A.-A. A. Jucis (1980). Tournaments and generalized Young tableaux (Russian). Mat. Zametki 27 353-359. MR0570745

[6] A. Kirillov (2000). Introduction to tropical combinatorics (English summary). Physics and Combinatorics 82-150. World Sci. Publishing. MR1872253

[7] S.-J. Kang, M. Kashiwara, K. C. Misra, T. Miwa, T. Nakashima, and A. Nakayashi (1991). Affine crystals and vertex models. Adv. Ser. Math. Phys. 16. World Sci. Publishing. MR1187560 
[8] S.-J. Kang, M. Kashiwara, K. C. Misra, T. Miwa, T. Nakashima, and A. Nakayashi (1992). Perfect crystals of quantum affine Lie algebras. Duke Math. J. 68 499-607. MR1194953

[9] A. Kirillov, A. Kuniba, and T. Nakanishi (1998). Skew Young diagram method in spectral decomposition of integrable lattice models. II. Higher levels. Nuclear Phys. B 529 611-638. MR1651921

[10] T. Lam and P. Pylyavskyy (2012). Total positivity in loop groups I: whirls and curls. Adv. in Math. 230 1222-1271. MR2921179

[11] T. Lam (2012). Loop symmetric functions and factorizing matrix polynomials. AMS/IP Stud. Adv. Math. 609-627. MR2908095

[12] A. Nakayashiki and Y. Yamada (1997). Kostka polynomials and energy functions in solvable lattice models. Selecta Mathematica 3 547-600. MR1613527

[13] M. Noumi and Y. Yamada (1999). Affine Weyl group symmetry in Painleve hierarchies. Painleve Systems, Hypergeometric Systems and Asymptotic Analysis. Kyoto. MR1778329

[14] M. Okado, A. Schilling, and M. Shimozono (2003). Virtual crystals and fermionic formulas of type $D_{n+1}^{(2)}, A_{2 n}^{(2)}$, and $C_{n}^{(1)}$. Represent. Theory 7 101-163. MR1973369

[15] M. Shimozono (2003). Crystals for dummies. http://www.aimath.org/ WWN/kostka/crysdumb.pdf.

[16] R. Stanley (2001). Enumerative Combinatorics, Volume 2. Cambridge Studies in Advanced Mathematics 62. Cambridge University Press. MR1676282

[17] Y. Yamada (2000). A birational representation of Weyl group, combinatorial $R$-matrix and discrete Toda equation. Physics and Combinatorics 305-319. World Sci. Publishing. MR1872262

THOMAS LAM

Department OF MATHEMATICS

UNIVERSITY OF MiCHIGAN

Ann Arbor

MI 48109

USA

E-mail address: tfylam@umich.edu 


\author{
Pavlo Pylyavskyy \\ Department of Mathematics \\ University OF MinNesota \\ MinNeAPOLIS \\ MN 55455 \\ USA \\ E-mail address: ppylyavs@umn.edu
}

Received May 28, 2013 